\title{
Avaliação, Monitoramento e Melhoria da Qualidade na APS
}

\author{
Elaine Tomasi, Fúlvio Borges Nedel, Allan Claudius Queiroz Barbosa
}

\section{RESUMO}

Esta nota técnica se insere no conjunto de ações desenvolvidas pela Rede de Pesquisa em Atenção Primária à Saúde e apresenta sua posição sobre avaliação, monitoramento e melhoria da qualidade na APS. Em um contexto de grave crise sanitária que desnuda as dificuldades institucionais, financeiras e operativas de resposta à situação de saúde, situa o debate e traz proposições que fortalecem uma prática avaliativa como instrumento essencial para o aprimoramento e fortalecimento de ações que potencializam o papel da APS no contexto do SUS. Estas notas abordam: a avaliação no contexto da APS em uma perspectiva histórica e institucional que situa sua relevância na própria trajetória do SUS. A importância de compreender os conceitos, métricas e indicadores e apropriá-los ao debate qualificado no âmbito da APS. Uma posição para a Rede APS em termos de desafios e proposições, visando a uma APS mais forte que nunca.

Palavras-chave: APS, Avaliação, Monitoramento, Qualidade.

\section{ABSTRACT}

This technical report is part of the set of actions developed by the Primary Health Care Research Network and presents its position on evaluation, monitoring and quality improvement in PHC. In a context of serious health crisis that exposes the institutional, financial and operational difficulties in responding to the health situation, it situates the debate and brings proposals that strengthen an evaluative practice as an essential instrument for the improvement and strengthening of actions that enhance the role of PHC in the context of the SUS. These notes address: the assessment in the context of PHC in a historical and institutional perspective that locates its relevance in the trajectory of the SUS. The importance of understanding the concepts, metrics and indicators and appropriating them to the qualified debate within the PHC. A position for the APS Network in terms of challenges and propositions, aiming for a stronger APS than ever.

Keywords: PHS, assessing, monitoring, quality.
Revista da Rede APS 2021

Publicada em: 20/09/2021

DOI:10.14295/aps.v3i2.208

\section{Elaine Tomasi}

(Universidade Federal de

Pelotas, Pelotas, RS, Brasil)

Fúlvio Borges Nedel

(Universidade Federal de Santa Catarina, Florianópolis, SC, Brasil)

Allan Claudius Queiroz Barbosa (Universidade Federal de Minas Gerais, Belo Horizonte, MG, Brasil)

Correspondência para:

Allan Claudius Queiroz Barbosa allan@ufmg.br 


\section{Apresentação: A Avaliação no CONTEXTO DA APS}

O monitoramento e a avaliação da qualidade na Atenção Primária à Saúde tem sido um tema relevante e de forte interesse de acadêmicos, gestores e profissionais desde a constituição do SUS. Com efeito, avaliações normativas de cariz governamental objetivaram institucionalizar o monitoramento e avaliação da APS. Some-se a isso a criação do Sistema de Informações da Atenção Básica (SIAB) em 1998, mecanismo de coleta de dados sobre cadastramento populacional e indicadores de atenção a usuários nos territórios ${ }^{1}$ (Bittar et al., 2009).

Em 1998, foi instituído o Pacto dos indicadores da Atenção Básica, regulamentado em 1999 como instrumento nacional de monitoramento e avaliação das ações e serviços de saúde referentes a esse nível de atenção, estabelecido entre as três esferas de gestão. Naquele momento, o Ministério da saúde reafirmava o caráter político e norteador do planejamento das ações do setor (Brasil, 2004). Entre 1999 e 2002, o processo do Pacto foi aprimorado com a inclusão de indicadores mais sintéticos e estratégicos em relação às áreas prioritárias da atenção básica e a definição de indicadores principais e complementares, o que permitiu maior adequação da análise às diferentes realidades regionais e locais. Nesse processo, a participação da academia subsidiou fortemente a definição de indicadores, seus critérios e atributos.

No início dos anos 2000, os Estudos de Linha de Base (ELB) do Projeto de Apoio à Implantação e Consolidação do PSF no Brasil (BRASIL, 2001), posteriormente denominado Projeto de Expansão e Consolidação da Saúde da Família (Proesf), implantado em grandes centros urbanos, oportunizou a investigação de

\footnotetext{
${ }^{1}$ a este respeito ver https://aps.saude.gov.br/ape/siab

2 Um questionário enviado aos gestores municipais abordava os seguintes temas: perfil do secretário municipal de saúde, perfil do coordenador municipal do PSF, incentivos à implantação do PSF, trajetória de implantação do PSF, características gerais do funcionamento do PSF no município, organização e gestão, modelo de atenção à saúde
}

diferentes aspectos relacionados à qualidade da atenção, incluindo acesso e efetividade das ações de saúde. Os ELB se constituíram em um dos marcos da avaliação externa da rede de saúde no Brasil (Facchini et al., 2006; Hartz, Felisberto \& Silva, 2008). Os diferentes estudos propiciaram o conhecimento do funcionamento dos sistemas municipais de saúde implantados nos grandes municípios brasileiros, além do papel do PSF na (re)organização da atenção básica, visando a detectar obstáculos na condução desse processo e identificar indicadores de monitoramento da implantação do PSF nesses mesmos municípios².

Para subsidiar o desenvolvimento do Proesf, a pesquisa "Avaliação da Implementação do Programa Saúde da Família (PSF) em Grandes Centros Urbanos - Dez Estudos de Caso" foi elaborada pelo Nupes/Daps/Ensp/Fiocruz, cuja equipe técnica selecionou os municípios a serem pesquisados, assim como indicou aspectos e variáveis a serem incluídos no projeto de investigação. A amostra de municípios incluiu situações diversificadas de implantação do PSF de forma a detectar o maior número de fatores que facilitavam e dificultavam o processo (BRASIL, 2005).

Medina et al., (2018) destacaram a expressiva produção científica oriunda desses dados. A mesma revisão de literatura apontou os principais temas de pesquisa, e um dos destaques foi o conjunto de artigos sobre avaliação da efetividade da ESF e sobre a qualidade do cuidado a grupos vulneráveis ou a agravos específicos.

Além dos já conhecidos estudos do efeito da cobertura populacional da ESF sobre tendências em indicadores de morbi-mortalidade (Macinko, Guanais e Souza, 2006; Macinko et al.., 2007; Aquino, Oliveira e Barreto, 2008; Moura et al., 2010; Rasella, Aquino e Barreto, 2010; Macinko

e relação com a comunidade, recursos humanos, financiamento e gastos, elementos facilitadores e dificultadores à implantação do PSF e expectativas do gestor e impactos do PSF. 
et al., 2011; Dourado et al., 2011; Rasella et al., 2014; Ceccon, Meneguel \& Viecili, 2014), devem-se mencionar estudos dedicados a indicadores de processo com foco em ações programáticas típicas da $A B$.

É importante situar ainda que as avaliações normativas no contexto da APS tiveram um significativo avanço quando o Ministério da Saúde realizou, entre 2001 e 2002, amplo estudo nacional, onde avaliou o processo de implantação do Programa Saúde da Família (PSF), focando a infraestrutura das unidades, gestão e processo de trabalho das equipes à luz dos princípios e diretrizes do PSF no país. 0 estudo incluiu mais de treze mil equipes de saúde da família e mais de 2.500 equipes de saúde bucal, percorrendo cerca de $70 \%$ dos municípios brasileiros para colher dados detalhados sobre a situação existente naquele momento, o que resultou em relatório publicado em 2004 (BRASIL, 2004b).

Com efeito, a partir de $2003^{3}$, a criação da Coordenação Geral de Acompanhamento e Avaliação da Atenção Básica (CGAA) do Ministério da Saúde gerou um espaço privilegiado de ações coordenadas, seja em parceria com instituições seja por meio de estudos conduzidos internamente pelo MS.

Ainda, o aprimoramento e a validação em vários países do Primary Care Assessment Tool (PCATool) teve no Brasil sua validação feita por Harzheim et al. (2006). A partir dos atributos essenciais e derivados da APS propostos por Starfield (2002), o PCATool considera características da estrutura e do processo de trabalho das equipes, inspirado no modelo de

\footnotetext{
${ }^{3}$ É importante situar a realização em 2003 do VII Congresso Brasileiro de Saúde Coletiva, onde aconteceu a Oficina "Avaliação da Atenção Básica - Estratégias de Institucionalização", com técnicos do Ministério da Saúde, CONASS e CONASEMS, além de pesquisadores e profissionais de saúde. Seu objetivo foi elaborar estratégias que pudessem contribuir para a integração, racionalização e institucionalização dos processos de avaliação da atenção básica. Ao final, foram ressaltados os seguintes eixos/dimensões do processo de avaliação: universalidade, integralidade, participação social, resolubilidade, acessibilidade aos diferentes níveis do sistema e equidade, considerando que a equidade deve ser um eixo transversal,
}

Donabedian (1966) para avaliação de serviços de saúde.

Diferentes estudos promoveram o debate acerca de resultados e desempenho no âmbito da APS. Barbosa et al (2006). Por exemplo, desenvolveram uma metodologia de avaliação de desempenho a partir de dados obtidos de cinco municípios mineiros, abrangendo 132 equipes e 3.522 usuários. Nesse estudo, foi proposto um escore que refletisse o cumprimento dos diversos princípios para cada uma das equipes avaliadas e para o município de forma global. Os resultados evidenciaram uma lacuna no que se referia à realização do processo de trabalho e um déficit de infra-estrutura disponível para dar suporte às atividades das equipes.

Posteriormente, em 2008, com a necessidade de atualização e monitoramento da situação existente, foi realizado estudo amostral que preservou aspectos metodológicos de 2001/2002 e, ao mesmo tempo, assegurou comparabilidade evolutiva nas dimensões avaliadas à época. Por meio de dados coletados junto a 3992 equipes em 564 municípios brasileiros, permitiu à então Diretoria de Atenção à Saúde do Ministério da Saúde um retrato cristalino da situação existente (Barbosa, 2008).

Estudo realizado em 2009 destinou-se a avaliar o acesso e a utilização de serviços de saúde, com ênfase no desempenho dos serviços e na qualidade dos cuidados de saúde, incluindo a APS, em cem municípios de pequeno, médio e grande porte populacional das cinco regiões geopolíticas do Brasil.4

que perpassa todos os demais. Apontou-se para a necessidade de considerar como eixo a integração institucional no âmbito do MS e como dimensões as práticas e a avaliação do contexto. Sugeriu-se, também, a elaboração de uma matriz cruzando as dimensões com os eixos estratégicos da Norma Operacional da Assistência à Saúde (NOAS).

4 https://dms.ufpel.edu.br/aquares/aquares/ 
Em 2010, a CGAA do Departamento de Atenção Básica do Ministério da Saúde institui a Avaliação para Melhoria da Qualidade (AMQ). Baseada em uma metodologia de autoavaliação e autogestão, tinha a finalidade de estreitar a relação entre os campos da avaliação e da qualidade, possibilitando aos atores diretamente envolvidos com o fazer em saúde seja no plano gerencial ou assistencial - a apropriação de métodos e ferramentas tanto para a realização de um diagnóstico acerca da organização e do funcionamento dos serviços e práticas como para a construção de projetos de intervenção sobre os desafios identificados (BRASIL, 2010).

A partir de 2011, novas diretrizes para a avaliação da qualidade da atenção básica começaram a ser discutidas e aprofundadas com a participação de diversas entidades acadêmicas e da sociedade civil, culminando na criação do Programa de Melhoria do Acesso e da Qualidade da Atenção Básica - PMAQ-AB (Portaria no 1.654, de 19 de julho de 2011, que institui o PMAQ-AB (BRASIL, 2012; 2012b). O PMAQ-AB teve como objetivo induzir a ampliação do acesso e a melhoria da qualidade da $A B$ por meio de processos contínuos e progressivos de monitoramento e avaliação, operacionalizados por repasses financeiros a municípios de acordo com o desempenho das equipes incluídas no Programa.

É importante observar que a transferência de recursos intergestores tais como incentivo para a melhoria da qualidade foi a estratégia adotada para fortalecer a atenção primária à saúde com a implantação do Programa. O incentivo financeiro, denominado Componente de Qualidade do Piso de Atenção Básica Variável (PAB Variável), era transferido fundo a fundo aos municípios com equipes que aderiram ao Programa. É notável, no processo de Avaliação Externa do PMAQ, a mencionada participação da sociedade civil e instituições acadêmicas de ensino e pesquisa. Frente à opção de se encomendar uma pesquisa ao IBGE (e sem detrimento do importantíssimo trabalho realizado por essa instituição), o MS resolve que a pesquisa deve ser construída pela opinião plural da sociedade e da academia e levada a cabo por instituições públicas de ensino e pesquisa com tal expertise.

Em 2019, a nova gestão federal instituiu o Programa PREVINE Brasil (BRASIL, 2019), em substituição ao PMAQ. Esse novo programa gerou um retrocesso no paradigma de financiamento da $A B$ ao centrar o incentivo no cadastro individual e no alcance de metas de um pequeno número de indicadores selecionados, sem definição de território de abrangência e população de referência das equipes de saúde. (DE SETA, M. H.; OCKÉ-REIS, C.O.; RAMOS, 2021)

Facchini, Tomasi e Dilélio (2018), em ensaio sobre a qualidade da APS no Brasil, apontaram avanços, desafios e perspectivas. Os autores destacaram a necessidade de um conjunto de indicadores de qualidade que expressem processos organizacionais e práticas profissionais de cada equipe da ESF na rede de serviços de saúde e na relação com o território. Postulam que a qualidade da atenção e, em consequência, a integralidade se explicitam, por exemplo, no alcance da completude ou totalidade de ações previstas em documentos oficiais para cada condição de saúde. Essa medida implica estimar a integração das ações previstas, incluindo a promoção da saúde, a prevenção de doenças, o tratamento e a reabilitação.

Também deve ser destacada, neste breve histórico sobre as últimas décadas, a preocupação em institucionalizar iniciativas de avaliação dos serviços de saúde ofertados no cotidiano da gestão da APS, tendo o território como núcleo organizacional e utilizando-se de uma extensa gama de indicadores. Isso naturalmente decorre do reconhecimento da saúde como direito de cidadania e associa-se à necessidade de atendimento da população com eficácia e qualidade por meio de uma estratégia diferenciada de saúde, reduzindo custos no longo prazo (SILVA, RIBEIRO e SILVEIRA, 2004; KLOETZEL et al., 1998). Ainda, o desenvolvimento desses estudos e de muitos outros atesta o longo período em que, de forma crescente, a política e as ações de APS foram embasadas em evidências produzidas em conjunto entre o SUS/APS e a academia. 
Para isso, torna-se necessário compreender e disponibilizar ações e ferramentas de fortalecimento de uma oferta de serviços de saúde que seja efetivamente vinculada à lógica do SUS, em consonância com o que o usuário deve e merece ter acesso. A Figura 1 sistematiza os principais momentos avaliativos realizados ao longo das últimas décadas no Brasil desde a criação do SUS.

Figura 1 - Marcos da Avaliação Normativa no Brasil - 1988 a 2019

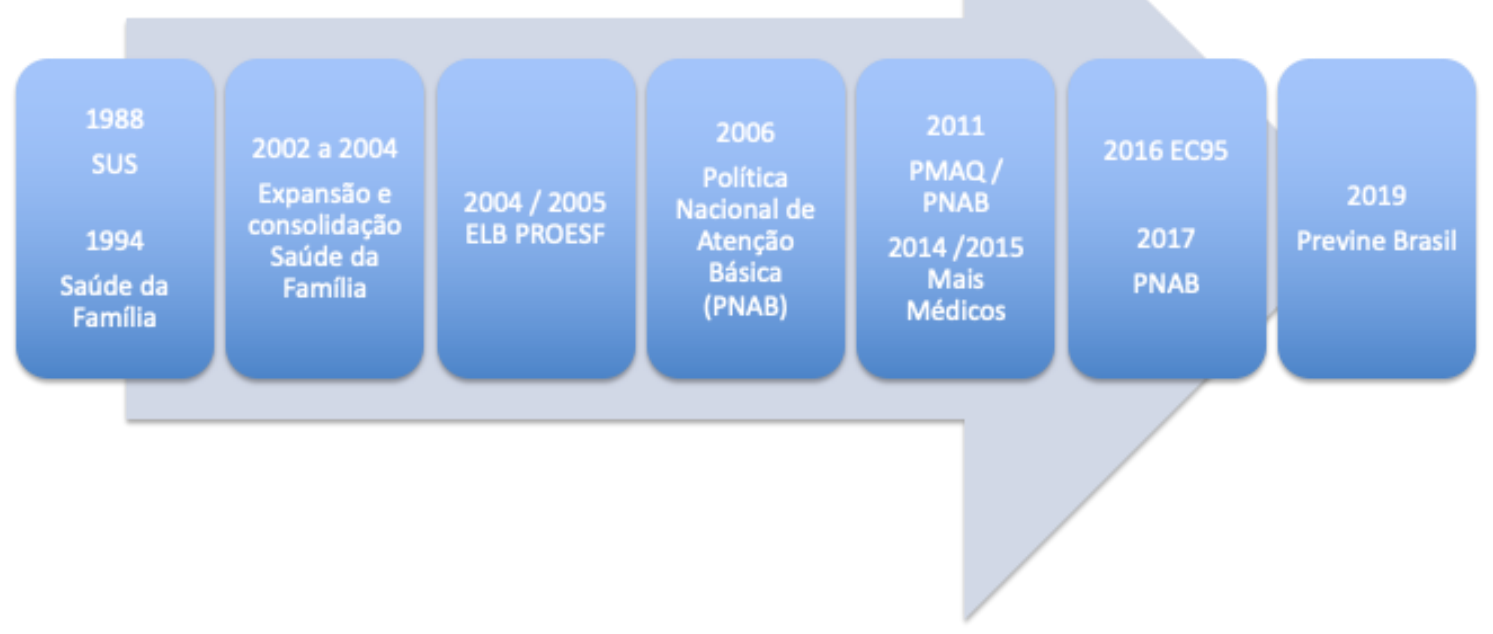

Fonte: Elaborada pelos autores.

De forma geral, conforme indicava em 2018 a Rede APS,

A realização de avaliações normativas do Programa de Saúde da Família, a criação da Coordenação Geral de Acompanhamento e Avaliação da Atenção Básica (CGAA) em 2003, os Estudos de Linha de Base do Proesf em 2005, a proposta de Avaliação para Melhoria da Qualidade (AMQ) da ESF, seguida pelo PMAQ-AB e a Autoavaliação o para Melhoria do Acesso e Qualidade da Atenção Básica (Amaq-AB), representam esforços relevantes do monitoramento $e$ avaliação da $A B$ no país. (Rede de Pesquisa em Atenção Primária à Saúde da Abrasco, 2018, pág. 418)

Neste breve histórico, há que se reconhecer o importante papel de diferentes atores - da academia, dos serviços e da sociedade civil - e suas contribuições basilares no aprimoramento dos processos de monitoramento e avaliação da APS, notadamente relacionadas a princípios, técnicas e instrumentos (RIBEIRO \& SCATENA, 2019). Os grandes estudos realizados influenciaram a tomada de decisão na gestão pública da saúde no Brasil, além de fortalecer diferentes espaços de ensino e pesquisa com relevante produção científica. Hoje, há um divórcio entre a gestão do SUS e a academia que impossibilita uma ação sistêmica e permite observar claramente que os importantes resultados obtidos daquela frutífera parceria institucional foram descontinuados no atual cenário político nacional.

\section{COMO APROPRIAR CONCEITOS,} MÉTRICAS E INDICADORES AO DEBATE DA APS

Uma das questões centrais deste debate e prática na saúde parte de quais atributos de 
avaliação devem ser levados em consideração e da percepção dos usuários sobre as práticas adotadas. Com efeito, quando uma oferta de serviços de saúde é feita com qualidade, certamente seus efeitos sobre a satisfação dos usuários e a qualidade percebida tornam-se visíveis.

Genericamente a satisfação está ligada à qualidade do produto ou serviço ofertado percebida por quem o recebe. Por isso, parte-se inicialmente do mapeamento daquelas necessidades. Dantas (2001), ao observar a perspectiva do cliente, afirma que a satisfação do cliente será percebida quando a organização ofertante demonstrar empenho de fato em descobrir o que ele necessitava ao desenvolver um produto ou serviço adequado. Berry e Parasuraman (1992) observam que, quando essa oferta acontece de forma inadequada, os usuários, denominados clientes pelos autores,

$$
\begin{aligned}
& \text { prestam mais atenção ao } \\
& \text { desempenho da companhia quando } \\
& \text { algo dá errado do que quando tudo } \\
& \text { funciona bem. Serviços com falhas } \\
& \text { produzem uma emoção mais intensa } \\
& \text { e, assim, uma avaliação mais } \\
& \text { carregada da parte do cliente do que } \\
& \text { o serviço impecável (BERRY e } \\
& \text { PARASURAMAN, 1992, p. 53) }
\end{aligned}
$$

Classicamente, quando se fala em qualidade de serviços, a ideia corrente é a de que sua medição se dá pelo matching entre expectativas do cliente e o nível de serviço a ele entregue. Isso deveria balizar, inclusive, diretrizes estratégicas nas relações entre sociedade e os prestadores de bens ou serviços.

Quando se fala da APS e sua interação direta com a comunidade, a premissa adotada por Kloetzel et al. (1998) deve balizar o debate, qual seja, a satisfação do usuário como sendo um indicador essencial na avaliação da qualidade dos serviços prestados. A característica de localidade e capilaridade da APS, isto é, o trabalho da equipe de saúde sobre pequenos contingentes populacionais e a maior dificuldade para garantir a confiabilidade de registros rotineiros em ações tão variadas e com tantas unidades registradoras limitam consideravelmente a seleção de indicadores. Há que se considerar ainda o nível de gestão da política ou ação que se avalia, desde a equipe de saúde local até a gestão nacional da APS.

Assim, permanece na APS o processo de territorialização ou "diagnóstico comunitário", isto é, a descrição das características da população sobre a qual se trabalha: sua forma de viver, características gerais e particulares de sua cultura, necessidades em saúde e equipamentos e fortalezas para seu enfrentamento (Kroeger \& Luna, 1987). Tais características são construídas historicamente em processos sociais de diferente duração e se materializam no território (Gondim \& Monken, 2018).

A territorialização é feita ao se iniciar o trabalho sobre uma população - implantação de uma nova Unidade Básica ou equipe de Saúde - e periodicamente, para atualizar a informação por meio de dados secundários e primários, coletados ad hoc. Outros níveis de gestão usarão esses dados agregados ou se beneficiarão de outros que, em nível local, têm menor precisão por exigirem denominadores populacionais maiores, como os de mortalidade e de internação hospitalar. Os principais indicadores utilizados na avaliação e monitoramento da APS podem ser sistematizados conforme o Quadro 1 a seguir. 
Quadro 1 - Indicadores frequentes utilizados na avaliação e monitoramento da APS.

\begin{tabular}{|c|c|c|c|c|}
\hline \multicolumn{2}{|r|}{ Atributo / Indicador } & Fonte & Nível de gestão & Acesso \\
\hline \multirow{10}{*}{$\begin{array}{l}\text { Estrutura e } \\
\text { processos de } \\
\text { trabalho }\end{array}$} & $\begin{array}{l}\text { Estrutura predial e ambiência da } \\
\text { Unidade de Saúde }\end{array}$ & AE-PMAQ & $\begin{array}{l}\text { Equipe de saúde, } \\
\text { Município, Estado, } \\
\text { União }\end{array}$ & DATASUS \\
\hline & Insumos e equipamentos & AE-PMAQ & $\begin{array}{l}\text { Equipe de saúde, } \\
\text { Município, Estado, } \\
\text { União }\end{array}$ & DATASUS \\
\hline & $\begin{array}{l}\text { № de estabelecimentos e } \\
\text { profissionais de saúde }\end{array}$ & CNES & $\begin{array}{l}\text { Município, Estado, } \\
\text { União }\end{array}$ & DATASUS \\
\hline & $\begin{array}{l}\text { № de equipes de Saúde da } \\
\text { Família }\end{array}$ & CNES, SIAB & $\begin{array}{l}\text { Município, Estado, } \\
\text { União }\end{array}$ & DATASUS \\
\hline & $\begin{array}{l}\text { № de pessoas atendidas em } \\
\text { diferentes condições de saúde } \\
\text { (pré-natal, puericultura, } \\
\text { condições crônicas, ...) }\end{array}$ & SIAB & $\begin{array}{l}\text { Equipe de saúde, } \\
\text { Município, Estado, } \\
\text { União }\end{array}$ & $\begin{array}{l}\text { Registros da } \\
\text { equipe de saúde } \\
\text { da família; } \\
\text { DATASUS }\end{array}$ \\
\hline & $\begin{array}{l}\text { № de visitas domiciliares por } \\
\text { equipe ou trabalhador de saúde }\end{array}$ & SIAB & $\begin{array}{l}\text { Equipe de saúde, } \\
\text { Município, Estado, } \\
\text { União }\end{array}$ & $\begin{array}{l}\text { Registros da } \\
\text { equipe de saúde } \\
\text { da família; } \\
\text { DATASUS }\end{array}$ \\
\hline & $\begin{array}{l}\text { Cobertura e concentração } \\
\text { vacinal }\end{array}$ & SI-PNI & $\begin{array}{l}\text { Equipe de saúde, } \\
\text { Município, Estado, } \\
\text { União }\end{array}$ & $\begin{array}{l}\text { Registros na } \\
\text { Unidade de Saúde; } \\
\text { DATASUS }\end{array}$ \\
\hline & $\begin{array}{l}\% \text { Gestantes com VDRL e HIV } \\
\text { solicitados }\end{array}$ & SINASC & $\begin{array}{l}\text { Equipe de saúde, } \\
\text { Município, Estado, } \\
\text { União }\end{array}$ & $\begin{array}{l}\text { Registros na } \\
\text { Unidade de Saúde; } \\
\text { DATASUS }\end{array}$ \\
\hline & $\begin{array}{l}\% \text { Diabéticos com exame do pé } \\
\text { realizado }\end{array}$ & AE-PMAQ & $\begin{array}{l}\text { Equipe de saúde, } \\
\text { Município, Estado, } \\
\text { União }\end{array}$ & DATASUS \\
\hline & Indicadores do PCATool & Coleta ad hoc & $\begin{array}{l}\text { Equipe de saúde, } \\
\text { município }\end{array}$ & -- \\
\hline \multirow{4}{*}{ Resultados } & $\begin{array}{l}\text { \% de consultas médicas não } \\
\text { encaminhadas a outros níveis }\end{array}$ & SIAB & $\begin{array}{l}\text { Equipe de saúde, } \\
\text { Município, Estado, } \\
\text { União }\end{array}$ & $\begin{array}{l}\text { Registros da } \\
\text { equipe de saúde } \\
\text { da família; } \\
\text { DATASUS }\end{array}$ \\
\hline & $\begin{array}{l}\text { Taxas de hospitalização por } \\
\text { diferentes condições de saúde } \\
\text { (diarréia em crianças, } \\
\text { pneumonia em idosos, } \\
\text { hipertensão, diabete, ...) }\end{array}$ & SIH/SUS & $\begin{array}{l}\text { Município, Estado, } \\
\text { União }\end{array}$ & DATASUS \\
\hline & Taxa de mortalidade infantil & SIM; SINASC & $\begin{array}{l}\text { Municípios grandes, } \\
\text { Estado, União }\end{array}$ & DATASUS \\
\hline & $\%$ e Taxa de ICSAP & $\mathrm{SIH} / \mathrm{SUS}$ & $\begin{array}{l}\text { Município, Estado, } \\
\text { União }\end{array}$ & DATASUS \\
\hline
\end{tabular}

\section{Fonte: elaborado pelos autores}

Notas: AE-PMAQ: Avaliação Externa do Programa de Melhoria e Avaliação da Qualidade da Atenção Básica CNES: Cadastro Nacional de Estabelecimentos de Saúde

DATASUS: Departamento de Informática do SUS

SIAB: Sistema de Informação da Atenção Básica

PCATool: Primary Care Assessment Tool

SIH/SUS: Sistema de Informações Hospitalares do SUS

SIM: Sistema de Informações sobre Mortalidade

SINASC: Sistema de Informações sobre Nascidos Vivos

ICSAP: Internações por Condições Sensíveis à Atenção Primária 
Dentre os indicadores apresentados no Quadro 1 , vale destacar, por exemplo, os resultados da aplicação do PCATool e as ICSAP, por serem indicadores compostos, que procuram apresentar uma medida integrada dos diferentes aspectos da APS.

O PCATool é um questionário aplicado à equipe de APS sobre diferentes aspectos de estrutura de processo de trabalho, organizados conceitualmente segundo os atributos da APS propostos por Starfield. O resultado das medidas classifica a equipe de saúde quanto ao alcance de cada um dos atributos e se possui qualidade na sua validade conceitual e empírica para medida dos atributos analisados (Harhzheim et al, 2018). Sua limitação metodológica está na dificuldade de aplicação mesmo em sua versão reduzida, o que o torna pouco útil para o monitoramento.

A ICSAP, por sua vez, é um indicador da capacidade da APS de resolver os problemas de saúde cuja atenção é de sua responsabilidade, antes que o paciente necessite uma internação hospitalar para o problema. É um indicador indireto da qualidade da APS, medido por eventos hospitalares. É composto por um amplo leque de causas que envolve 19 grupos da Lista Brasileira de Condições Sensíveis à Atenção Primária, abarcando, em grande parte, a diversidade da clínica em APS, além de contar com dados mais confiáveis e de divulgação mais atualizada ${ }^{5}$.

A avaliação e monitoramento na APS enfrenta desafios pelo amplo escopo de seu objeto. A amplitude da prática na APS, porta de entrada do usuário no sistema de saúde, além de seu papel de coordenação da atenção aos casos que necessitam encaminhamento a outras instâncias do sistema (Starfield, 2002), torna a APS responsável por atender a todas as questões de saúde, ainda que com pequeno protagonismo numa pequena proporção dos

\footnotetext{
${ }^{5}$ Sua tabulação on-line via TABNET não está disponibilizada, mas pode ser feita pelo TabWin ou por meio de um pacote no programa $\mathrm{R}$, de acesso livre, sendo muito útil para o monitoramento da APS. Sua principal limitação está em apenas apontar falhas na APS e em que tipos de doenças ou população essas falhas se concentram, mas não em que
}

casos. Daí sua magnitude e a relevância dos mecanismos e práticas consistentes de ação avaliativa voltadas à melhoria das condições de saúde da população.

No âmbito do PMAQ-AB, eram avaliados 47 indicadores de sete áreas estratégicas e classificados segundo a natureza de seu uso. Os indicadores de desempenho são vinculados ao processo de avaliação externa e utilizados para a classificação das equipes de saúde, ao passo que os indicadores de monitoramento precisam ser acompanhados de forma regular para complementação de informações sobre a oferta de serviços e resultados alcançados por cada equipe, sem, no entanto, influenciar na pontuação atribuída às $E A B$ no processo de avaliação externa.

Já o programa Previne Brasil, a partir de 2019 (Brasil, 2019), estabeleceu um elenco reduzido de indicadores que, além de seu escopo limitado, impõe uma questão fundamental para a gestão: como estimar a cobertura em cada equipe sem os conceitos de território e população de referência, quando o sistema de saúde é universal? No SUS, usar a população cadastrada como denominador de metas para a $A B$ só pode ser aceito se o cadastramento for um censo populacional. De outra forma, as metas podem ser alcançadas com a exclusão de uma parcela da população, o que, felizmente, não permite a Constituição Federal. A universalidade do SUS exige que as políticas e serviços de saúde - e, portanto, seus indicadores - tenham como população-alvo a totalidade da população residente num determinado território. Sem um denominador de base populacional para cada serviço, é impossível estimar seu alcance sobre a cidadania. (Massuda, 2019)

\section{UMA POSIÇÃO PARA A REDE APS}

Agora, mais do que nunca, é preciso fortalecer a APS em seus imperativos de qualidade nos

ponto do sistema de saúde (estrutura, o processo de trabalho, gestão da Unidade de Saúde ou do Município, etc.), a falha se localiza. (Mendonça et al., 2018) 
serviços prestados. Os desafios permanecem e precisam ser superados:

- O que medir para avaliar a qualidade da APS?

- Quais indicadores e tipologia devem ser utilizados?

- É possível assegurar o monitoramento contínuo e análises pontuais da APS?

- Como realizar de forma robusta e a custos baixos a pesquisa de campo?

- Quais bases de dados podem auxiliar?

- Como ajustar a produção e o tratamento da informação considerando inadequação de registros e sistemas de informação?

- É possível compatibilizar os sistemas de informação em saúde do subsistema privado ao e-SUS?

- Como garantir tratamento adequado e divulgação transparente e precisa da informação?

Há ainda uma série de outros desafios que merecem ser destacados com vistas à melhoria da qualidade e uso da informação para a gestão da saúde na APS:

- A garantia da realização de censos, contagens populacionais e pesquisas nacionais sobre as condições de vida e saúde da população brasileira;

- A oferta atualizada de estimativas populacionais por sexo e faixa etária para os municípios, com disponibilização das bases de dados no DATASUS e não apenas a possibilidade de tabulação on-line;

- O aumento e a qualificação de trabalhadores da vigilância em saúde nas secretarias municipais e estaduais de saúde;

- A qualidade, a transparência e o acesso aos dados devem melhorar, o que envolve, pelo menos:

- o investimento para a melhoria da informação sociodemográfica nos SIS existentes;
- a disponibilização da informação para tabulação on-line e download de dados do subsistema de saúde suplementar;

- a organização, via política nacional, dos SIS da AB nos municípios, para garantir a disponibilidade de informação nos mesmos moldes do DATASUS;

- maior harmonização dos registros, padronização no processo de coleta de dados e interoperabilidade entre os SIS;

- a organização de painéis on-line ("dashboards", a exemplo do que temos visto para os dados da Covid19) para a informação ao público sobre a situação de saúde no seu município. O TABNET é muito útil para o trabalhador de saúde (capacitado para seu uso), mas de pouco apelo para o cidadão comum ou para um conselheiro municipal de saúde sem essa capacitação.

Além da necessária geração de insumos e informações capazes de assegurar uma oferta qualificada, é fundamental, acima de tudo,

I -promoção da universalidade, integralidade e equidade na atenção e proteção à saúde, direcionada à continuidade do cuidado individual e coletivo por meio dos processos de coleta, gestão, produção e disseminação dos dados e informação em saúde;

II - fomento à gestão e à produção dos dados e informação em saúde, como elementos capazes de gerar conhecimento, na totalidade das ações de atenção, gestão, auditoria, pesquisa, controle e participação social, de modo a fundamentar ações 
de vigilância em saúde e formulação de políticas públicas. ${ }^{6}$

Os intentos estratégicos que a Rede APS forjou em 2018 permanecem atuais:

Desenvolver estratégias para a melhoria da qualidade do cuidado individual e coletivo.

Incorporar as novas tecnologias de informação e comunicação, contribuindo para a melhor resolutividade da APS, ampliação do acesso e formação dos trabalhadores.

Aprofundar os estudos sobre acesso e qualidade na APS, com vistas a garantir a universalização da ESF e a integralidade das ações na rede básica de saúde, com ênfase na redução de desigualdades sociais e regionais.

Desenvolver e implementar estratégias metodológicas para mensurar e estimar o impacto das intervenções em APS.

Estabelecer um sistema de monitoramento das iniquidades em saúde e discutir os desafios da avaliação de impacto das ações intersetoriais sobre a saúde.

Explorar e divulgar resultados das avaliações externas do PMAQ-AB, de modo a compor um painel complexo sobre padrões e tendências na $A B$ brasileira, em apoio à gestão e aos trabalhadores em saúde. (Rede APS, 2018)

Faz-se necessária, portanto, a existência de um Sistema de Informação em Saúde (SIS) que registre com validade e precisão os diferentes aspectos envolvidos na organização e prática da APS. A grande diversidade de sistemas - Sistema de Informações sobre Mortalidade/SIM, Sistema de Informação sobre Nascidos

\footnotetext{
${ }^{6} \mathrm{~A}$ este respeito ver

http://www.susconecta.org.br/cns-aprovapolitica-nacional-de-informacao-e-informaticaem-saude/
}

Vivos/SINASC, Sistema de Informações Hospitalares do SUS/SIH/SUS, Sistema de Informações sobre Agravos de Notificação/SINAN, Sistema de Informação do Programa Nacional de Imunizações/SI-PNI, dentre outros - traz consigo dificuldades, pois são concebidos, organizados e alimentados com lógicas diversas e sem um identificador unívoco que permita o acompanhamento do indivíduo em sua relação com o Sistema de Saúde ao longo da vida ou a união de bases dos diferentes SIS (Moreno, 2018).

Não obstante tais dificuldades, merecem destaque algumas forças, como a disponibilidade de dados dos principais SIS e a possibilidade de tabulações on-line por meio da ferramenta TABNET do DATASUS ou de tabulações mais detalhadas ou definidas pelo usuário por meio do software TabWin, também do DATASUS.

Ainda, é fundamental assegurar que o vasto esforço empreendido pela comunidade da APS, seja ela inserida nos espaços acadêmicos seja atuando na gestão ou na "ponta" da oferta de serviços, em oferecer estudos, pesquisas e relatos de experiências de natureza avaliativa, não se perca. Os estudos devem ser disponibilizados para consulta e análise, sob pena de tornar sua produção descontínua e desatualizada, sem condições de compreender a dinâmica social. Por fim, é importante ressaltar que a avaliação e o monitoramento não devem ser tratados como eventos episódicos. Pelo contrário, são processos dinâmicos e em contínuo aperfeiçoamento e devem embasar a busca constante pela qualidade na Atenção Primária à Saúde e no Sistema Único de Saúde. 


\section{REFERÊNCIAS}

AQUINO, R; OLIVEIRA, N.F.; BARRETO, M.L. Impact of the Family Health Program on Infant Mortality in Brazilian Municipalities. American Journal of Public Health (1971), v. 99, p. 87-93, 2008.

BARBOSA, A. C. Q. Proposição de Metodologia de Avaliação de Resultados e Desempenho das Unidades Básicas e Satisfação dos Usuários de Atenção Primária à Saúde. Um desenho elaborado a partir de Cidades de Minas Gerais. Belo Horizonte: UFMG/CNPq, 2006. ISBN 978-85-60500-03-1 (estudo realizado através do Edital MCT/CNPq/MS-SCTIE-DECIT no 023/2006).

BARBOSA, A. C. Q. (Coordenação Geral) Saúde da família no Brasil: situação atual e perspectivas - Estudo Amostral 2008 / Avaliação Normativa do Programa Saúde da Família no Brasil - Monitoramento da Implantação das Equipes de Saúde da Família e Saúde Bucal. Brasília: DAB/UFMG, 2008 (relatório final)

BERRY, L. L.; PARASURAMAN, A. Serviços de marketing: competindo através da qualidade. São Paulo: Maltese-Norma, 1992.

BITTAR, T. O. et al. O sistema de informação da atenção básica como ferramenta da gestão em saúde. RFO, Passo Fundo, RS, v. 14, n. 1, p. 77-81, jan./abr. 2009. Disponível em: <http://www.upf.br/download/editora/revistas/rfo/14-01/77_81.pdf>

BRASIL. Ministério da Saúde, Secretaria de Políticas de Saúde, Departamento de Atenção Básica. Projeto de apoio à implantação e consolidação do Programa de Saúde da Família no Brasil. Brasília, 2001. Mimeo.

BRASIL. Ministério da Saúde. Secretaria de Atenção à Saúde. Departamento de Atenção Básica. Monitoramento na atenção básica de saúde: roteiros para reflexão e ação / Secretaria de Atenção à Saúde, Departamento de Atenção Básica. - Brasília: Ministério da Saúde, 2004. 72 p.: il.-(Série C. Projetos, Programas e Relatórios).

BRASIL. Ministério da Saúde. Avaliação normativa do Programa Saúde da Família no Brasil: monitoramento da implantação e funcionamento das equipes de saúde da família: 2001-2002. Brasília: $\begin{array}{lllll}\text { Ministério da } & \text { Saúde: 2004b. Available }\end{array}$ http://bvsms.saude.gov.br/bvs/publicacoes/avaliacao normativa programa saude familia.pdf.

BRASIL. Ministério da Saúde. Fundação Oswaldo Cruz. Saúde da Família: avaliação da implementação em dez grandes centros urbanos: síntese dos principais resultados / Ministério da Saúde, Fundação Oswaldo Cruz; [elaborado por Sarah Escorel (Coord.); Lígia Giovanella; Maria Helena Mendonça; Rosana Magalhães; Mônica de Castro Maia Senna]. - 2. ed. atual. - Brasília: Editora do Ministério da Saúde, 2005. 210 p.: il. color.- (Série C. Projetos, Programas e Relatórios)

BRASIL. Ministério da Saúde. Melhoria contínua da qualidade na atenção primária à saúde: conceitos, métodos e diretrizes. Brasília: Ministério da Saúde, 2010. (Série B. Textos Básicos de Saúde).

BRASIL. Ministério da Saúde. PORTARIA № 1.654, DE 19 DE JULHO DE 2011. Institui, no âmbito do Sistema Único de Saúde, o Programa Nacional de Melhoria do Acesso e da Qualidade da Atenção Básica (PMAQ$A B)$ e o Incentivo Financeiro do PMAQ-AB, denominado Componente de Qualidade do Piso de Atenção Básica Variável - PAB Variável. Brasília; 2012.

BRASIL. Ministério da Saúde. Secretaria de Atenção à Saúde. Departamento de Atenção Básica. Programa de Melhoria do Acesso e da Qualidade da Atenção Básica. Brasília; 2012(b).http://189.28.128.100/dab/docs/publicacoes/geral/manual instrutivo pmaq site.pdf. 
BRASIL, Ministério da Saúde. Portaria 2979 de 12/11/2019. Institui o Programa Previne Brasil. http://189.28.128.100/dab/docs/portaldab/documentos/financiamento/portarias/prt 2979121120 19.pdf

CECCON, R. F.; MENEGHEL, S.N. \& VIECILLI, P.R.N. Hospitalization due to conditions sensitive to primary care and expansion of the Family Health Program in Brazil: an ecological study. Revista Brasileira de Epidemiologia, v. 17, p. 968-977, 2014

DANTAS, E. B. Satisfação do cliente: um conforto entre a teoria, o discurso e a prática. Dissertação de mestrado. Programa de Pós-graduação em Engenharia de Produção, Universidade Federal de Santa Catarina, 2001.

DE SETA, M. H.; OCKÉ-REIS, C.O.; RAMOS, A.L.P. Programa Previne Brasil: o ápice das ameaças à Atenção Primária à Saúde? Ciênc. saúde coletiva 26 (suppl 2) 30 Ago 2021.

DONABEDIAN, A. Evaluation the quality of medical care. Milbank Q. 1966; 44:166.

DOURADO, I. et al. Trends in primary health care-sensitive conditions in Brazil: the role of the Family Health Program (Project ICSAP-Brazil). Medical Care, 49(6): 577-584, 2011.

FACCHINI, L. A. et al. Desempenho do PSF no Sul e no Nordeste do Brasil: avaliação institucional e epidemiológica da atenção básica à saúde. Ciência \& Saúde Coletiva, Rio de Janeiro, v. 11, p. 669-681, 2006.

FACCHINI, L. A.; TOMASI, E.; DILÉLIO, A.S. Qualidade da Atenção Primária à Saúde no

Brasil: avanços, desafios e perspectivas. Saúde em Debate | Rio De Janeiro, V. 42, Número Especial 1, P. 208-223, setembro, 2018.

GONDIM, G.M.M. \& MONKEN, M. O uso do território na Atenção Primária à Saúde. In: Mendonça, M.H.M. et al. (orgs). Atenção primária à saúde no Brasil: conceitos, práticas e pesquisa. Rio de Janeiro. Ed. Fiocruz, 2018.

HARTZ, Z.M.A.; FELISBERTO, E.; SILVA, L.M.V. Meta-Avaliação da Atenção Básica à Saúde: teoria e prática. Rio de Janeiro. Editora FIOCRUZ. ISBN: 978-85-7541-162-9. 2008.

HARZHEIM, E. et al. Consistência interna e confiabilidade da versão em português do Instrumento de Avaliação da Atenção Primária (PCATool-Brasil) para serviços de saúde infantil. Cadernos de Saúde Pública (ENSP. Impresso), v. 22, p. 1649-1659, 2006.

HARZHEIM, E. et al. Estudos de PCATool no Brasil. In: Mendonça, M.H.M. et al. (orgs). Atenção primária à saúde no Brasil: conceitos, práticas e pesquisa. Rio de Janeiro. Ed. Fiocruz, 2018.

KLOETZEL K et al. Controle de qualidade em atenção primária à saúde I - A satisfação do usuário. Cadernos de Saúde Pública, Rio de Janeiro 14 ( 3), jul/set. pp. 623-628, 1998.

KROEGER, A. \& LUNA, R. 1987. Atención primaria de salud: principios y métodos. Organización Panamericana de la Salud. ISBN 968-860-416-X. https://iris.paho.org/handle/10665.2/3101.

MACINKO, J.; GUANAIS, F.C. \& SOUZA, M.F. Evaluation of the impact of the Family Health Program on infant mortality in Brazil, 1990-2002. Journal of Epidemiology \& Community Health, 60(1):13-19, 2006.

MACINKO, J. et al. Going to scale with community=based primary care: an analysis of the Family Health Program and infant mortality inn Brazil, 1999-2004. Social Science \& Medicine, 65(10):2.070-2.080, 2007. 
MACINKO, J. et al. The Influence of Primary Care and Hospital Supply on Ambulatory Care-Sensitive Hospitalizations Among Adults in Brazil, 1999-2007. American Journal of Public Health (1971), v. 1, p. e1e8, 2011.

MASSUDA, A. Mudanças no financiamento da Atenção Primária à Saúde no Sistema de Saúde Brasileiro: avanço ou retrocesso? Ciênc. saúde coletiva 25 (4); Mar 2020.

MEDINA, M.G. et al. A pesquisa em atenção primária à saúde no Brasil. In: Mendonça, M.H.M. et al. (orgs). Atenção primária à saúde no Brasil: conceitos, práticas e pesquisa. Rio de Janeiro. Ed. Fiocruz, 2018.

MENDONÇA, C. S. et al. A utilização do indicador Internações por Condições Sensíveis à Atenção Primária no Brasil. In: Mendonça, M.H.M. et al. (orgs). Atenção primária à saúde no Brasil: conceitos, práticas e pesquisa. Rio de Janeiro. Ed. Fiocruz, 2018.

MORENO, A. Muito além do básico: as bases de dados em saúde e a atenção básica à saúde no Brasil. In Mendonça, M.H.M. et al. (orgs). Atenção primária à saúde no Brasil: conceitos, práticas e pesquisa. Rio de Janeiro. Ed. Fiocruz, 2018.

MOURA, B.L.A. et al. Principais causas de internação por condições sensíveis à Atenção Primária em Saúde no Brasil: uma análise por faixa etária e região. Revista Brasileira de Saúde Materno-Infantil, 10(S1):8391, 2010.

RASELLA, D. et al. Impact of primary health care on mortality from heart and cerebrovascular diseases in Brazil: a nationwide analysis of longitudinal data. BMJ, v. 349, p. g4014-g4014, 2014.

REDE DE PESQUISA EM ATENÇÃO PRIMÁRIA À SAÚDE DA ABRASCO. Contribuição para uma agenda política estratégica para a Atenção Primária à Saúde no SUS. Saúde debate 42(spe1), set 2018. https://doi.org/10.1590/0103-11042018S128.

RIBEIRO, L.A., SCATENA, J.H. A avaliação da atenção primária à saúde no contexto brasileiro: uma análise da produção científica entre 2007 e 2017. Saúde Soc. São Paulo, v.28, n.2, p.95-110, 2019.

SILVA, Jane Azevedo; RIBEIRO, Luiz Cláudio; SILVEIRA, Márcio. Avaliação do nível de satisfação dos usuários do Programa Saúde da Família no Bairro Furtado de Menezes - Juiz de Fora: [s.I.], 2004.

STARFIELD, B. Atenção primária: equilíbrio entre necessidades de saúde, serviços e tecnologia / Bárbara Starfield. Brasília : UNESCO, Ministério da Saúde, 2002. 726p. 